Research Article

\title{
Investigation of the Morphological, Structural, and Vibrational Behaviour of Graphite Nanoplatelets
}

\author{
H. M. Albetran \\ Department of Basic Sciences, College of Education, Imam Abdulrahman Bin Faisal University, Dammam 31451, Saudi Arabia \\ Correspondence should be addressed to H. M. Albetran; halbatran@iau.edu.sa
}

Received 16 January 2021; Revised 23 May 2021; Accepted 24 May 2021; Published 11 June 2021

Academic Editor: Jim Low

Copyright (c) 2021 H. M. Albetran. This is an open access article distributed under the Creative Commons Attribution License, which permits unrestricted use, distribution, and reproduction in any medium, provided the original work is properly cited.

\begin{abstract}
This work investigated the morphological, structural, and vibrational properties of graphite nanoplatelets (GNPs) that were produced by ultrasonication. Scanning and transmission electron microscopy, X-ray diffractometry, and Raman spectroscopy identified 120-nm-thick GNP crystallites and 50-2000 $\mu \mathrm{m}^{2}$ plates with different areas and shapes. Extensive exfoliation was observed by transmission electron microscopy with abundant multi and some monolayer GNPs. X-ray diffractometry confirmed 43 GNP layers along the $c$-axis. Rietveld X-ray analysis indicated a GNP crystal lattice with stacks of parallel two-dimensional graphene layers and tightly bound hybridized carbon atoms stacked in a translational ...ABAB... sequence in hexagonal rings. Raman scattering indicated well-defined GNPs with few defects and no oxide content. All analytical results reveal that GNPs could have significant potential application in electrically conductive reinforcement devices.
\end{abstract}

\section{Introduction}

Carbon can catenate with elemental carbon or other elements to form organic structures as the basis of life. Zero-, one-, two-, and three-dimensional structures, which are termed fullerenes, carbon nanotubes, graphene, and graphite, respectively, are basic elemental carbon-based structures $[1,2]$.

Graphite is one of the oldest known forms of carbon and is termed black lead or plumbago. In Greek, "grapho" means "to write" and graphite is used extensively in pencils and lubricants. Graphite occurs in metamorphic rocks (sedimentary carbon-compound reduction during metamorphism), in igneous rocks, and in meteorites [3]. Graphite is used in electronic products, such as batteries, electrodes, and solar panels, because of its high conductivity, and low cost. Its high thermal conductivity and low mass make it an ideal thermal conductive polymer composite in manufacturing [3-7].

Despite graphite appearing to be a well-defined homogeneous product with a known chemical and physical structure, different properties are displayed by different graphite types (amorphous, flake, vein, and synthetic). The two forms or phases of graphite include rhombohedral and hexagonal crystal structures with similar physical properties (density $=1.9 \mathrm{~g} / \mathrm{cm}^{3}$, iron-black to steel-grey colour, and deep-blue in transmitted light). The hexagonal and rhombohedral graphite phases have a $\mathrm{P}_{63} / \mathrm{mcc}$ and $\mathrm{R}_{3} \mathrm{~m}$ space group, four and six atoms per unit cell (Z), and $a=0.2461 \mathrm{~nm}$ and $c=0.6708 \mathrm{~nm}$ and $a=0.2456 \mathrm{~nm}$ and $c=0.6696 \mathrm{~nm}$ lattice parameters at room temperature, respectively $[8,9]$. The single-crystal lattice stacking periodicity structure of the hexagonal $(2 \mathrm{H})$ and rhombohedral $(3 \mathrm{R})$ graphite phases is $\mathrm{ABAB}$ and $\mathrm{ABCABC}$, respectively. The graphite particle size is readily reduced by mechanical grinding because the interlayer bonding force is weaker than that of the intralayers [10-16]. Graphene, which is the term for individual graphite layers, can be restacked to prepare graphite [17]. In the stacking, carbon atoms are layered in 0.142-nm-spaced honeycomb lattices, and planes are separated by $0.335 \mathrm{~nm}$ [5]. Their similar structures prevent characterization techniques from being used to distinguish between graphite and graphene [18].

Graphite nanomaterials are of interest because of their safe, affordable, stable, nontoxic, noncarcinogenic, bactericidal, photocatalytic, industrial, biomedical, and wastewater treatment applications [7, 19]. Carbon nanostructures can be produced from graphite, such as three-dimensional 
carbon nanotubes and vapor-grown carbon nanofibers from the coiling of covalent graphite building [20], diamond-like $\mathrm{sp}^{3}$, and fullerene-like bonding from ball milling for up to $1000 \mathrm{~h}$ in vacuum [15]. Structural changes are of scientific importance with wide potential for application [11-16], and graphite nanoplatelet graphitic nanofillers (GNPs, also termed graphite nanosheets, graphite nanoflakes, or exfoliated or expanded graphite) from randomly stacked two-dimensional graphene sheets [4]. The incorporation of graphene/graphite flakes into polymer composites increases their thermal conductivity [21-23].

Many methods exist to prepare GNPs with several layers, quantities, and qualities. These methods include mechanical cleavage, thermal exfoliation of acid-intercalated graphite, high-pressure homogenization, micromechanical exfoliation of graphite, chemical vapor deposition, chemical reduction of graphene oxide, electrochemical exfoliation, vacuum filtration, liquid-phase exfoliation, and ultrasonication [24-29]. Among the fabrication methods, ultrasonication-assisted liquid-phase exfoliation is simple, yields GNPs large quantities, and is a cost-effective technique that is applicable at industrial levels to fabricate GNPs.

In this study, scanning electron microscopy (SEM) and transmission electron microscopy (TEM), X-ray diffractometry (XRD, with Rietveld refinement), and Raman spectroscopy were used to determine the physicochemical properties of GNPs that were synthesized using ultrasonic exfoliation of flake graphite in liquid.

\section{Experimental Methodology}

2.1. Material Synthesis. Graphite flakes (molecular weight $12.01 \mathrm{~g} / \mathrm{mol}$, product number 332461, Sigma-Aldrich) were used to prepare the GNPs by immersion in $70 \%$ isopropyl alcohol and $30 \%$ water with ultrasonication at $40 \mathrm{kHz}$ in a bath for $5 \mathrm{~h}$ at room temperature, which resulted in shear forces and cavitation by micron-sized bubble growth and collapse. GNPs were formed by rinsing and calcining the product dispersion at $250^{\circ} \mathrm{C}$. Figure 1 shows the ultrasonication steps for GNPs.

\subsection{Materials Characterization}

2.2.1. SEM. The GNP morphology was studied by scanning electron microscopy (SEM) using IRMC-INSPECT S50 microscopy. The GNPs were not coated to prevent charging because they are a good electrical conductor.

2.2.2. TEM. Ground samples of GNPs $(\sim 5 \mathrm{mg})$ were ultrasonicated in an ethanol suspension for $10 \mathrm{~min}$. The transmission electron microscope (TEM) imaging (FEI MorgagniTM 268, tungsten tip electron source) was carried out on copper grids with two drops of suspension.

2.2.3. XRD. The GNP purity and structure were confirmed by XRD using the "Rigaku Benchtop Miniflex X-ray" diffractometer $\left(\lambda_{\mathrm{Cu}-\mathrm{K} \alpha}=0.1541 \mathrm{~nm}\right)$. The spectra were in the $2 \theta$ region of $20-70^{\circ}$ with a scanning speed of $0.02^{\circ} / \mathrm{min}$. Rietveld pattern analysis using FullProf software (version 7.20) was used with a goodness-of-fit from the weighted pattern R- factor $\left(R_{\mathrm{wp}}\right)$, derived Bragg R-factors $\left(R_{P}\right)$, and expected Rfactor $\left(R_{\exp }\right)$. A patterned background, optimized sample displacement, preferred orientation, peak shape, $2 \theta_{0}$, scale factor, and lattice parameters were used to calculate the GNP crystalline phase abundances. Rietveld refinements were undertaken by using the Crystallography Open Database and graphite crystal structures (COD 9000046).

2.2.4. Raman Spectroscopy. Raman spectroscopy provides structural and chemical knowledge of different materials based on information about the vibrational states of a compound. Photons moved by Raman have a Stokes or antiStokes diffusion. Because photons are bosons, the Stokes $\left(I_{\text {Stokes }}\right)$ and anti-Stokes intensities $\left(I_{\text {anti-Stokes }}\right)$ are linked to $(n+1)$ or $n$, respectively, such as $n$ is the Bose Einstein population factor. The $I_{\text {Stokes }}$ is more intense than the $I_{\text {anti-Stokes, }}$ which is usually evaluated and recorded in traditional Raman spectroscopy [30].

Because of the intensity of the materials during Raman scattering, some expressions for data processing are necessary as provided below. Stokes phenomenon represents phonon emissions, whereas the anti-Stokes phenomenon represents phonon absorption. The energy change during these processes is given by

$$
\begin{aligned}
\hbar \omega_{\text {Stokes }} & =\hbar \omega_{i}-\hbar \omega_{p}, \\
\hbar \omega_{\text {anti-Stokes }} & =\hbar \omega_{i}+\hbar \omega_{p},
\end{aligned}
$$

where $\omega_{p}$ is the angular frequency of the scattered phonon, $\hbar$ is the reduced Planck constant, $\omega_{\text {Stokes }}$ is the angular frequency of the scattered photon, and $\omega_{i}$ is the angular frequency of the incident photon. The intensities $I_{\text {anti-Stokes }}$ of the first-order scattering by a phonon/boson are given by

$$
\begin{aligned}
& I_{\text {Stokes }} \propto I_{i} \chi^{\prime \prime}(\omega) \omega_{s}^{3}[n(\omega, T)+1] \\
& I_{\text {anti-Stokes }} \propto I_{i} \chi^{\prime \prime}(\omega) \omega_{s}^{3}[n(\omega, T)] .
\end{aligned}
$$

The phonon $n(\omega, T)$ parameter at thermal equilibrium is given by

$$
n(\omega, T)=\frac{1}{\exp \left(\hbar \omega / k_{B} T\right)-1}=\frac{1}{\exp (1.439 \times \omega / T)-1},
$$

where $T$ is the absolute temperature and $k_{B}$ is the Boltzmann constant. Raman spectroscopy was undertaken on a LabRAM 1B dispersive Raman spectrometer. The GNPs were excited using a $633 \mathrm{~nm}$ line. A laser light was focused through a 50x objective and a 600 lines $/ \mathrm{mm}$ diffraction grating, Peltier-cooled charge-coupled device detector at $-40^{\circ} \mathrm{C}$, with a $60 \mathrm{~s}$ collection time and $10 \mathrm{~s}$ for silicon. The silicon peak correction was undertaken by shifting the Raman spectra by a 520.7 wavenumber.

\section{Results and Discussion}

3.1. GNP Microstructures. SEM and TEM were used to determine the GNP particle size, morphology, and microstructure. 


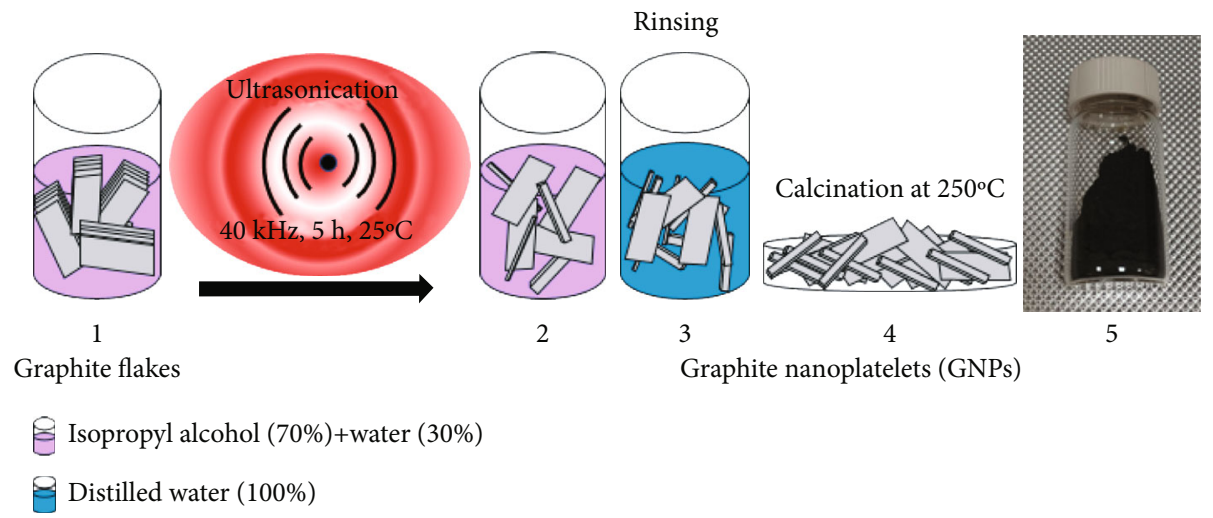

FIGURE 1: Schematic representation of GNP ultrasonication.

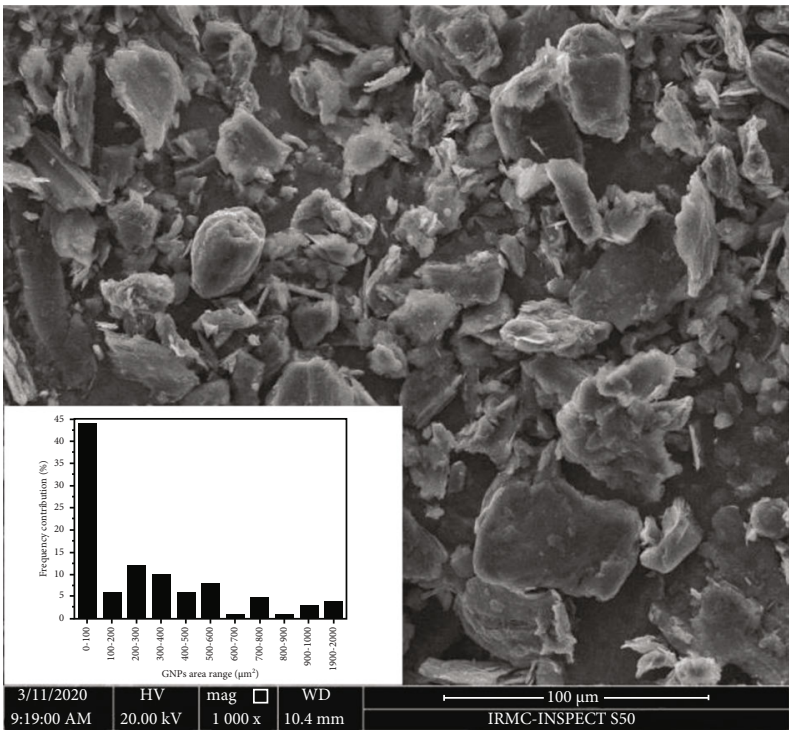

(a)

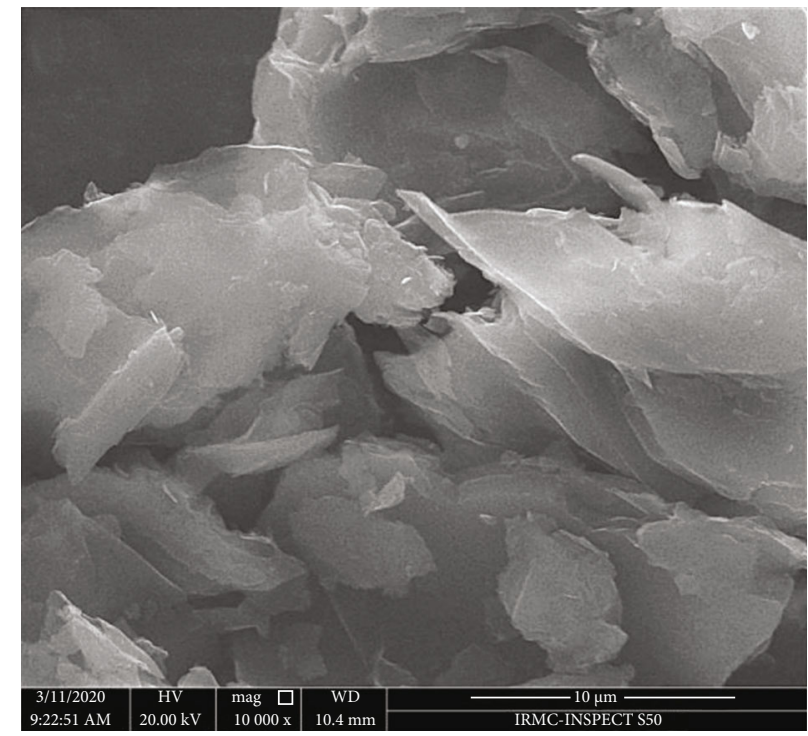

(b)

Figure 2: (a) Low- and (b) high-resolution SEM micrographs of GNPs.

Typical secondary electron images of sonicated graphite flakes are presented in Figures 2(a) and 2(b). Fragmented flake graphite yielded foliated parallelogram graphite plates. The area and thickness of one-hundred GNPs as determined by the ImageJ software were $50-2000 \mu \mathrm{m}^{2}$ and $\sim 120 \mathrm{~nm}$, respectively. These values were comparable with the nanosheets that Chen et al. obtained by ultrasonication of graphite powder [31].

The TEM micrographs in Figures 3(a) and 3(b) show graphite in nanoplatelets at a low resolution and granularity in the higher-resolution images, respectively. Nanoplatelets with varying shapes and areas formed because ultrasonication prior to TEM broke down graphite nanoplatelets into smaller pieces. The weak interlayer Van Der Waals attraction allowed the nanoplatelets to slide past each other perpendicular to the $c$-axis, but the sufficiently strong attraction prevented the complete formation of individual graphene layers [17]. The TEM diffraction contrast from thickness variations indicates that graphite nanoplatelets of a few layers, including bi- and tri-layers, formed, but some monolayer graphene was also visible. Polycrystalline graphite with randomly oriented crystallites and an interplanar spacing of $\sim 0.40 \mathrm{~nm}$ is shown in Figure 3(c). Single crystals in natural flake graphite were oriented in a preferred direction, whereas synthetic graphite was oriented more randomly [32].

3.2. $X R D$. Figure 4 shows the diffraction pattern of crystalline GNP structures. The hexagonal crystal structure of GNP with no impurity or second-phase peaks is shown by characteristic diffraction peaks that were indexed as (002), (020), (111), and (004) planes [11, 33].

The reflection profile broadening of the XRD pattern is used to calculate the crystallite size, and the corresponding peak position is used to determine the interlayer spacing. The average-crystallite size $\left(D_{\mathrm{XRD}}\right)$ of GNP was calculated from the Scherrer equation, and the $\mathrm{d}_{002}$ (d-spacing for $2 \mathrm{H}$ (002) from $2 \theta$ peak at $26.619^{\circ}$ ) was calculated from Bragg's Law $[33,34]$ 


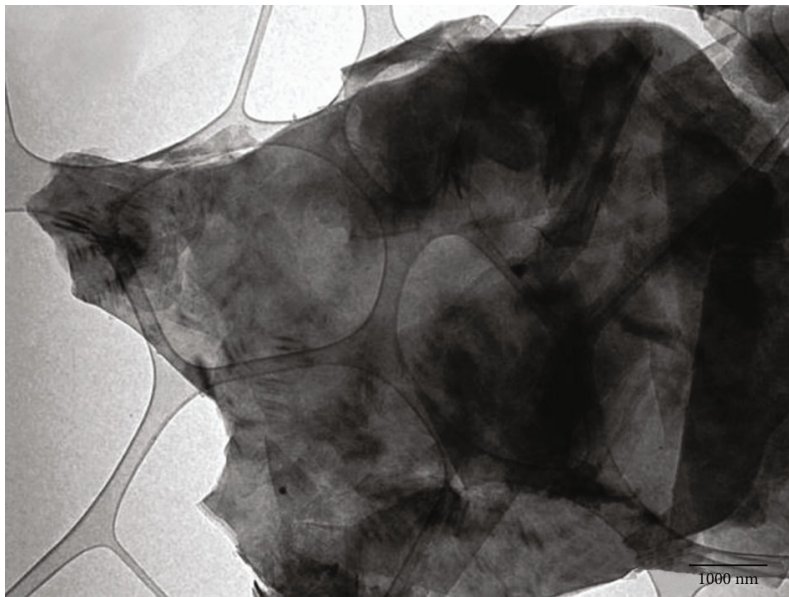

(a)

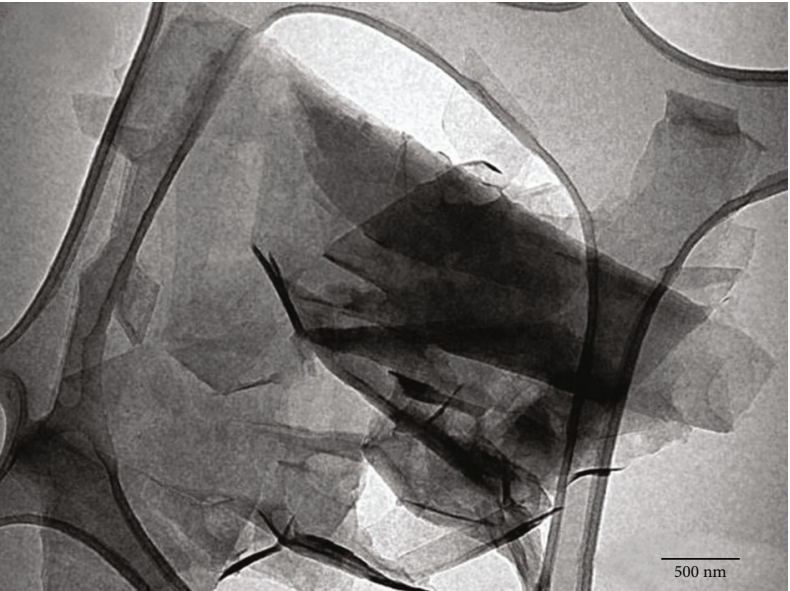

(b)

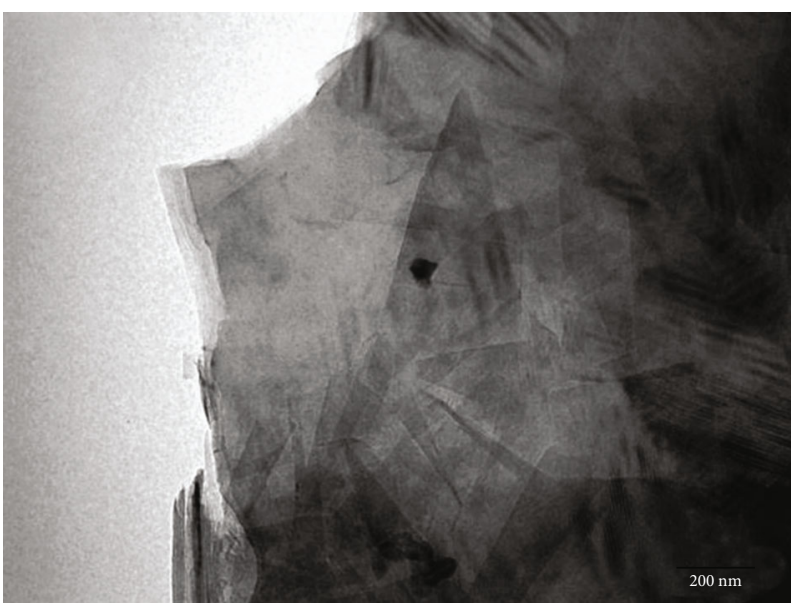

(c)

Figure 3: (a) Low-resolution and (b, c) lattice-resolved HR-TEM micrographs of GNPs with polycrystalline graphite material and $\sim 0.33627$ $\mathrm{nm}$ d-spacing between adjacent (002) lattice planes.

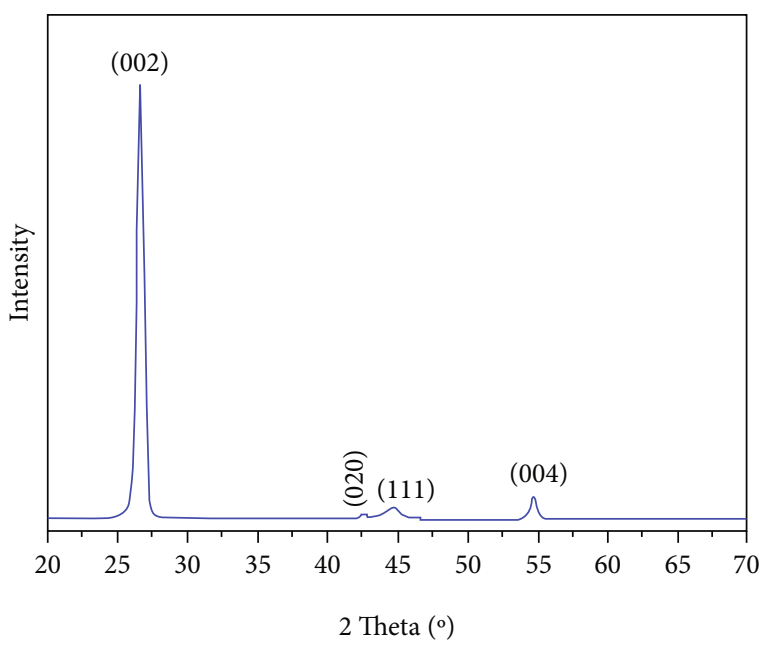

FIgURE 4: Room temperature XRD pattern of GNPs.

$$
\begin{aligned}
D_{\mathrm{XRD}} & =\frac{k \lambda}{\beta \cos \theta}, \\
d_{002} & =\frac{n \lambda}{2 \sin \theta},
\end{aligned}
$$

where $\theta, \beta, \lambda$, and $k$ are the Bragg diffraction angle, full width at half maximum (FWHM), wavelength of the $\mathrm{Cu}-\mathrm{K}_{\alpha}$ radiation $(0.15419 \mathrm{~nm})$, and shape factor $(0.91)$, respectively.

The computed crystallite size of $14.42 \mathrm{~nm}$ was compared well with the graphite sample sizes by Gen et al. [31]. The physical origins of the broad- (002) like reflections were interpreted from the uniform interlayer spacing $\left(\mathrm{d}_{002}\right)$ and are related directly to layer misalignment with average interlayer spacings, such as crystalline graphite $(0.335 \mathrm{~nm})$. These results agree with the TEM micrographs. Pure crystalline graphite has an identical basal spacing $[33,35]$.

Seehra et al. described the $N_{c}$ number of layers along the $c$ -axis as [36]

$$
N_{c}=\frac{D_{\mathrm{XRD}}}{d_{002}} .
$$




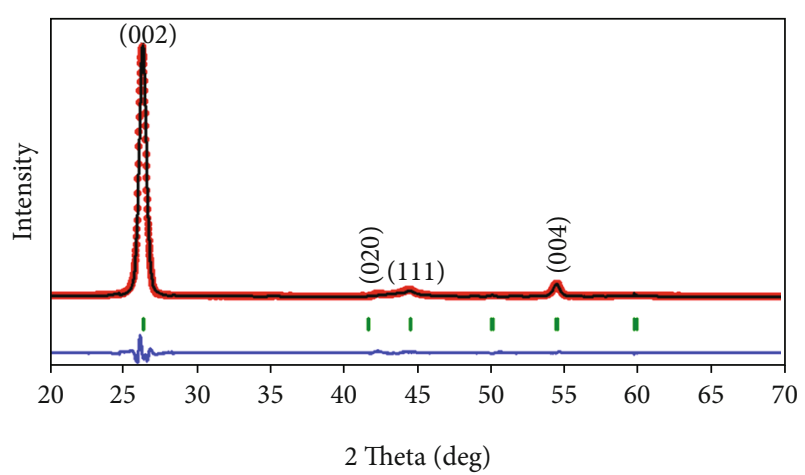

FIGURE 5: GNPs XRD Rietveld difference plots. Black cycles and the solid black line represent a measured and calculated pattern, respectively. Blue residual plot and green bars indicate the difference between the calculated and measured pattern and peak positions ((002), (020), and (004)), respectively.

They calculated the interlayer spacing $\left(\mathrm{d}_{002}\right)$ and apparent crystallite size $\left(D_{\mathrm{XRD}}\right)$ in the $c$-direction that provide the numbers of GNP layers. For this study, forty-three GNP layers exist along the $c$-axis.

3.3. XRD Rietveld Analysis. Rietveld pattern-fitting of the XRD pattern was analysed from the FullProf software, and the goodness-of-fit was estimated from the derived Bragg $\mathrm{R}$-factors $\left(R_{B}\right)$, expected R-factor $\left(R_{\exp }\right)$, and weighted pattern R-factor $\left(R_{\mathrm{wp}}\right)$ [37-43]. The preferred orientation, peak shape parameters, lattice parameters, scale factor, $2 \theta_{0}$, sample displacement, and pattern background were optimized in the Rietveld refinement to calculate the GNP lattice parameters. The residual GNP XRD Rietveld plots are shown in Figure 5 where the refinements were obtained from graphite crystal structures (COD 9000046). The solid black line and cycles indicate the calculated and measured pattern, respectively, with the difference between the two indicated by a blue residual plot. Green bars show the (002), (020), and (004) peak positions. In this work, the residual Rietveld refinements were 5.62 and $\sim 11.9$ for the $R_{\mathrm{wp}}$ and $R_{\text {exp }}$, respectively. The goodness-of-fit $\left(\chi^{2}\right)$ of 4.46 shows an acceptable refinement quality [37-43].

A comparison of the density, cell volume, and cell parameters of the GNPs with literature data is provided in Table 1. The calculated density of $2.28 \mathrm{~g} / \mathrm{cm}^{3}$, cell volume of 0.06996 $\mathrm{nm}^{3}$, and lattice parameters $a=0.24461 \mathrm{~nm}$ and $c=0.67254$ $\mathrm{nm}$ for the Rietveld hexagonal GNPs are smaller than the literature values $[8,11,44-48]$.

The existence of parallel 2D graphene layers with a translational ... $\mathrm{ABAB} \ldots$ sequence of tightly bonded $\mathrm{sp}^{2}$ hybridized carbon atoms in hexagonal rings is shown in Figure 6 from the Rietveld crystal lattice of GNP. The soft lubricating nature of the GNPs is provided by the covalently bonded carbon atoms in layers that are bound by weak Van Der Waals forces, which allows for graphene layer sliding. The distance between adjacent graphene layers in graphite $(0.336 \mathrm{~nm})$ is half the hexagonal graphite crystallographic spacing $(0.673$ $\mathrm{nm}$ ), which is like the calculated (002) peak d-spacing at $2 \theta$ of $26.619^{\circ}$.
TABLE 1: Comparison of hexagonal graphite lattice parameters, cell volume, and density with literature data.

\begin{tabular}{lccc}
\hline Study & $a(\mathrm{~nm})$ & $c(\mathrm{~nm})$ & $V\left(\mathrm{~nm}^{3}\right)^{*}$ \\
\hline This study & 0.24461 & 0.67254 & 0.06996 \\
{$[8]$} & 0.24560 & 0.66960 & 0.07081 \\
{$[44]$} & 0.24600 & 0.67100 & 0.07116 \\
{$[45]$} & 0.24620 & 0.67110 & 0.07133 \\
{$[46]$} & 0.24630 & 0.67120 & 0.07142 \\
{$[11,47]$} & 0.24612 & 0.67080 & 0.07126 \\
\hline
\end{tabular}

${ }^{*}$ Cell volume from XRD Rietveld analysis in this study and calculated from a hexagonal cylinder volume in other studies.

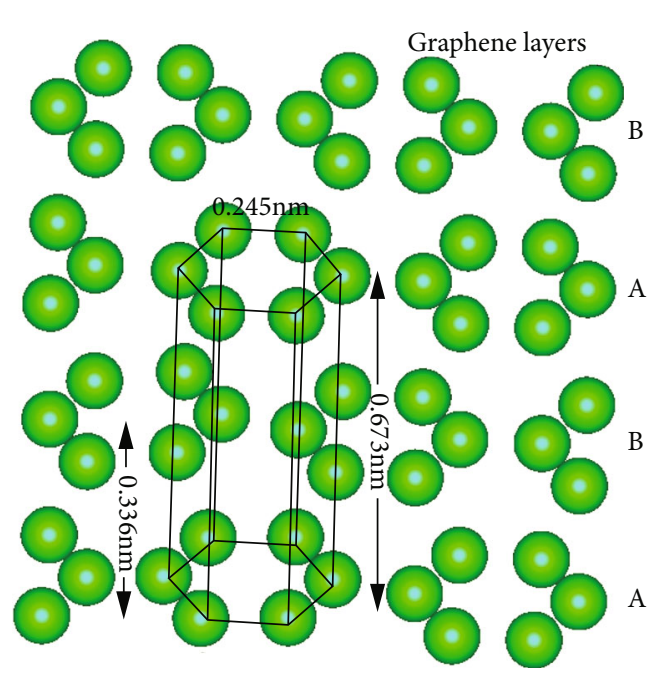

Figure 6: Hexagonal (2H) GNPs with graphene layers stacked in translational ...ABAB... sequence with room temperature 0.336 $\mathrm{nm}$ perpendicular interplanar distance.

3.4. Raman Spectroscopy. A GNP Raman spectrum in the range of $200-3200 \mathrm{~cm}^{-1}$ is shown in Figure 7 . The spectrum was plotted at a reduced intensity, and the measured intensity was divided by the Bose Einstein population factor $n(\omega)=$ $\left(e^{\hbar \omega / k T}\right)^{-1}$.

The G and two-dimensional (2D) band shape, position, and intensity were used to estimate the number of GNP layers. The $2 \mathrm{D}$ band changes position, width, and shape as the number of layers increases, but the peak position of the $\mathrm{G}$ band shifts downwards as the layer number increases. Characteristic GNP peaks exist in the D, 2D, and G bands at $1,331,2,686$, and $1,577 \mathrm{~cm}^{-1}$, respectively. A high sample quality is indicated by the weak and strong D and G peaks (for $\mathrm{sp}^{2}$ carbon), respectively, and the broad multiband 2D peak indicates multilayer graphite features [17]. A primary graphene band characteristic is illustrated by the $\mathrm{sp}^{2}$ carbon atom vibration in the $\mathrm{G}$ band. The $\mathrm{D}$ band provided a disordered GNP vibrational peak and was used to characterize GNP structural defects [42]. Well-defined GNPs with few defects are present. Raman-active defect concentrations in parts per million $(\mathrm{ppm})$ were calculated from the ratio of the defect density $\left(n_{D}\right)$ and number of carbon atoms $\left(n_{C}\right)[27,30]$ : 


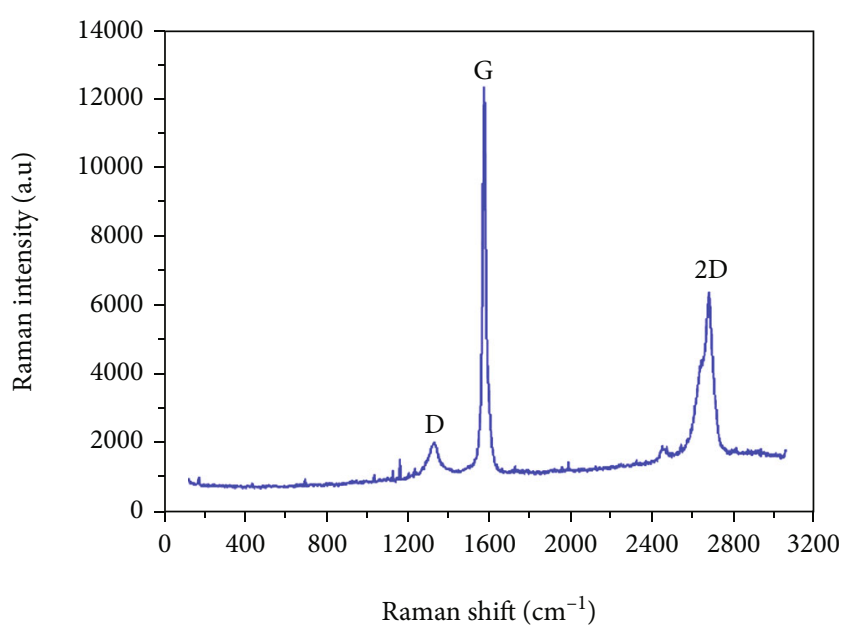

Figure 7: Raman spectrum after division by the Bose Einstein population factor of GNPs at room temperature and excited by a 633-nm laser line.

$$
\begin{aligned}
\frac{n_{D}}{n_{C}} & =\frac{2.16 \times 10^{11}}{3.82 \times 10^{15}} \frac{I(D)}{I(G)}, \\
n_{c} & =3.82 \times 10^{15} \mathrm{~cm}^{-2},
\end{aligned}
$$

where $I(D)$ and $I(G)$ are the D- and G-band integration area, respectively, and the concentration defects and integral area ratio are $\sim 0.18$ and $\sim 11 \mathrm{ppm}$, respectively. The measured $I(D) / I(G)$ ratio and GNP defect concentrations compared well with the literature values for samples of graphite and graphene [27]. These results confirm the XRD results (Figure 4) by the existence of parallel $2 \mathrm{D}$ graphene layers with a translational ...ABAB... sequence of tightly bonded $\mathrm{sp}^{2}$ hybridized carbon atoms in hexagonal rings. However, the investigation of this correlation between structural and vibrational proprieties proves that their nanocrystallite size, crystal system, and vibrational modes make GNPs good candidates for many practical applications in electrically conductive reinforcement devices.

\section{Conclusions}

Expanded-flake graphite was ultrasonicated in isopropyl alcohol to prepare GNPs. SEM, TEM XRD, and Raman spectroscopy were used to study the physicochemical properties of the GNPs. SEM and TEM indicated the formation of multi- and some monolayer parallelogram GNPs. The TEM diffraction contrast from thickness variations indicates that GNPs of a few layers, including bi- and tri-layers, formed, but some monolayer graphene was also visible. XRD analysis indicated the formation of 43 layers along the $c$-axis. The GNP crystal structure as determined by XRD and Rietveld refinement indicated nanometre-thick worm-like exfoliated graphite nanoplatelets. The $120-\mathrm{nm}$-thick graphite units from flaky graphite exfoliation provide a promising, lowcost, lightweight alternative to carbon- and metal-based electrically conductive reinforcement applications.

\section{Data Availability}

Data is available on request.

\section{Conflicts of Interest}

The author declares that there is no conflict of interest.

\section{Acknowledgments}

The author acknowledges financial support from the Imam Abdulrahman Bin Faisal University through the project application No. 2020-086-CED. Prof. It-Meng Low at the Department of Physics and Astronomy, Curtin University, Australia, is thanked for the manuscript review and feedback. The author would like to thank Prof. Abdulhadi Baykal, and Dr. Sultan Akhtar at Institute for Research and Medical (IRMC), Imam Abdulrahman Bin Faisal University (IAU), Dammam, Saudi Arabia, for assistance with materials characterization works.

\section{References}

[1] W. Choi, I. Lahiri, R. Seelaboyina, and Y. S. Kang, "Synthesis of graphene and its applications: a review," Critical Reviews in Solid State and Materials Sciences, vol. 35, no. 1, pp. 52-71, 2010.

[2] H. Kim, A. A. Abdala, and C. W. Macosko, "Graphene/polymer nanocomposites," Macromolecules, vol. 43, no. 16, pp. 6515-6530, 2010.

[3] R. Sengupta, M. Bhattacharya, S. Bandyopadhyay, and A. K. Bhowmick, "A review on the mechanical and electrical properties of graphite and modified graphite reinforced polymer composites," Progress in Polymer Science, vol. 36, no. 5, pp. 638-670, 2011.

[4] B. Li and W. H. Zhong, "Review on polymer/graphite nanoplatelet nanocomposites," Journal of Materials Science, vol. 46, no. 17, pp. 5595-5614, 2011.

[5] H. Tu and L. Ye, "Thermal conductive PS/graphite composites," Polymers for Advanced Technologies, vol. 20, no. 1, pp. 21-27, 2009.

[6] H. Fukushima, L. T. Drzal, B. Rook, and M. Rich, "Thermal conductivity of exfoliated graphite nanocomposites," Journal of Thermal Analysis and Calorimetry, vol. 85, no. 1, pp. 235238, 2006.

[7] M. Toyoda and M. Inagaki, "Heavy oil sorption using exfoliated graphite: new application of exfoliated graphite to protect heavy oil pollution," Carbon, vol. 38, no. 2, pp. 199-210, 2000.

[8] H. S. Lipson and A. Stokes, "The structure of graphite," Proceedings of the Royal Society of London. Series A. Mathematical and Physical Sciences, vol. 181, no. 984, pp. 101-105, 1942.

[9] H. Lipson and A. R. Stokes, "A new structure of carbon," Nature, vol. 149, no. 3777, p. 328, 1942.

[10] G. Bacon, "The determination of the unit-cell dimensions of non-cubic substances," Acta Crystallographica, vol. 1, no. 6, p. 337, 1948.

[11] Z. Li, C. Lu, Z. Xia, Y. Zhou, and Z. Luo, "X-ray diffraction patterns of graphite and turbostratic carbon," Carbon, vol. 45, no. 8, pp. 1686-1695, 2007.

[12] F. Salver-Disma, J. M. Tarascon, C. Clinard, and J. N. Rouzaud, "Transmission electron microscopy studies on carbon 
materials prepared by mechanical milling," Carbon, vol. 37, no. 12, pp. 1941-1959, 1999.

[13] T. Ong and H. Yang, "Effect of atmosphere on the mechanical milling of natural graphite," Carbon, vol. 38, no. 15, pp. 20772085, 2000.

[14] T. D. Shen, W. Q. Ge, K. Y. Wang et al., "Structural disorder and phase transformation in graphite produced by ball milling," Nanostructured Materials, vol. 7, no. 4, pp. 393-399, 1996.

[15] N. Welham, V. Berbenni, and P. Chapman, "Effect of extended ball milling on graphite," Journal of Alloys and Compounds, vol. 349, no. 1-2, pp. 255-263, 2003.

[16] P. Zhou, R. Lee, A. Claye, and J. E. Fischer, "Layer disorder in carbon anodes," Carbon, vol. 36, no. 12, pp. 1777-1781, 1998.

[17] W. Gu, W. Zhang, X. Li et al., "Graphene sheets from wormlike exfoliated graphite," Journal of Materials Chemistry, vol. 19, no. 21, pp. 3367-3369, 2009.

[18] P. Delhaes, Graphite and Precursors, CRC Press, 2000.

[19] G. Chen, W. Weng, D. Wu, and C. Wu, "PMMA/graphite nanosheets composite and its conducting properties," European Polymer Journal, vol. 39, no. 12, pp. 2329-2335, 2003.

[20] A. Ganguly, J. J. George, S. Kar, A. Bandyopadhyay, and A. K. Bhowmick, "Rubber nanocomposites based on miscellaneous nanofillers," in Current Topics in Elastomers Research, pp. 89-100, CRC Press, 2008.

[21] A. A. Balandin, "Phononics of graphene and related materials," ACS Nano, vol. 14, no. 5, pp. 5170-5178, 2020.

[22] F. Kargar, Z. Barani, R. Salgado et al., "Thermal percolation threshold and thermal properties of composites with high loading of graphene and boron nitride fillers," ACS Applied Materials \& Interfaces, vol. 10, no. 43, pp. 37555-37565, 2018.

[23] H. Malekpour, K. H. Chang, J. C. Chen et al., "Thermal conductivity of graphene laminate," Nano Letters, vol. 14, no. 9, pp. 5155-5161, 2014.

[24] D. G. Papageorgiou, I. A. Kinloch, and R. J. Young, "Mechanical properties of graphene and graphene-based nanocomposites," Progress in Materials Science, vol. 90, pp. 75-127, 2017.

[25] V. Vasanthi, S. Gayathri, K. Anitha, and V. Ramakrishnan, "Exfoliation of high-quality graphene in volatile and nonvolatile solvents," Graphene Technology, vol. 2, no. 1-2, pp. 29-40, 2017.

[26] Y. Xu, H. Cao, Y. Xue, B. Li, and W. Cai, "Liquid-phase exfoliation of graphene: an overview on exfoliation media, techniques, and challenges," Nanomaterials, vol. 8, no. 11, pp. 942-974, 2018.

[27] X. You, Q. Feng, J. Yang, K. Huang, J. Hu, and S. Dong, "Preparation of high concentration graphene dispersion with low boiling point solvents," Journal of Nanoparticle Research, vol. 21, no. 1, pp. 19-30, 2019.

[28] V. Guerra, C. Wan, V. Degirmenci et al., "Characterisation of graphite nanoplatelets (GNP) prepared at scale by highpressure homogenisation," Journal of Materials Chemistry C, vol. 7, no. 21, pp. 6383-6390, 2019.

[29] C. Backes, A. M. Abdelkader, C. Alonso et al., "Production and processing of graphene and related materials," $2 D$ Materials, vol. 7, article $022001,2020 \mathrm{http}: / /$ hdl.handle.net/10486/ 691025 .

[30] T. H. Kauffmann, N. Kokanyan, and M. D. Fontana, "Use of Stokes and anti-Stokes Raman scattering for new applications," Journal of Raman Spectroscopy, vol. 50, no. 3, pp. 418-424, 2019.
[31] G. Chen, W. Weng, D. Wu et al., "Preparation and characterization of graphite nanosheets from ultrasonic powdering technique," Carbon, vol. 42, no. 4, pp. 753-759, 2004.

[32] M. Wissler, "Graphite and carbon powders for electrochemical applications," Journal of Power Sources, vol. 156, no. 2, pp. 142-150, 2006.

[33] I. M. Low, H. M. Albetran, and M. Degiorgio, "Structural characterization of commercial graphite and graphene materials," Journal of Nanotechnology and Nanomaterials, vol. 1, no. 1, pp. 23-30, 2020.

[34] I. M. Low, H. Albetran, V. M. Prida, V. Vega, P. Manurung, and M. Ionescu, "A comparative study on crystallization behavior, phase stability, and binding energy in pure and Crdoped $\mathrm{TiO}_{2}$ nanotubes," Journal of Materials Research, vol. 28, no. 3, pp. 304-312, 2013.

[35] A. Yasmin and I. M. Daniel, "Mechanical and thermal properties of graphite platelet/epoxy composites," Polymer, vol. 45, no. 24, pp. 8211-8219, 2004.

[36] M. S. Seehra, U. K. Geddam, D. Schwegler-Berry, and A. B. Stefaniak, "Detection and quantification of $2 \mathrm{H}$ and $3 \mathrm{R}$ phases in commercial graphene-based materials," Carbon, vol. 95, pp. 818-823, 2015.

[37] H. Albetran and I. Low, "Crystallization kinetics study of Indoped and (In-Cr) co-doped $\mathrm{TiO}_{2}$ nanopowders using_in-situ_ high-temperature synchrotron radiation diffraction," Arabian Journal of Chemistry, vol. 13, no. 2, pp. 3946-3956, 2020.

[38] H. Albetran and I. M. Low, "Effect of indium ion implantation on crystallization kinetics and phase transformation of anodized titania nanotubes using in-situ high-temperature radiation diffraction," Journal of Materials Research, vol. 31, no. 11, pp. 1588-1595, 2016.

[39] H. Albetran, B. O'Connor, V. Prida, and I. Low, "Effect of vanadium ion implantation on the crystallization kinetics and phase transformation of electrospun $\mathrm{TiO}_{2}$ nanofibers," Applied Physics A: Materials Science \& Processing, vol. 120, no. 2, pp. 623-634, 2015.

[40] H. Albetran and I. M. Low, "Crystallization kinetics and phase transformations in aluminum ion-implanted electrospun $\mathrm{TiO}_{2}$ nanofibers," Applied Physics A: Materials Science \& Processing, vol. 122, no. 12, pp. 1044-1053, 2016.

[41] H. Albetran, V. Vega, V. M. Prida, and I. M. Low, "Dynamic diffraction studies on the crystallization, phase transformation, and activation energies in anodized titania nanotubes," Nanomaterials, vol. 8, no. 2, pp. 122-131, 2018.

[42] H. Albetran, H. Haroosh, Y. Dong, V. Prida, B. O'Connor, and I. M. Low, "Phase transformations and crystallization kinetics in electrospun $\mathrm{TiO}_{2}$ nanofibers in air and argon atmospheres," Applied Physics A: Materials Science \& Processing, vol. 116, no. 1, pp. 161-169, 2014.

[43] H. M. Albetran, “Thermal expansion coefficient determination of pure, doped, and co-doped anatase nanoparticles heated in sealed quartz capillaries using_in-situ_high-temperature synchrotron radiation diffraction," Heliyon, vol. 6, no. 7, article e04501, 2020.

[44] D. Chung, "Review graphite," Journal of Materials Science, vol. 37, no. 8, pp. 1475-1489, 2002.

[45] P. Trucano and R. Chen, "Structure of graphite by neutron diffraction," Nature, vol. 258, no. 5531, pp. 136-137, 1975.

[46] M. Mohr, J. Maultzsch, E. Dobardzic et al., "Phonon dispersion of graphite by inelastic X-ray scattering," Physical Review $B$, vol. 76, no. 3, article 035439, 2007. 
[47] Y. Hishiyama and M. Inagaki, "Lattice parameter changes in graphite with boron doping," Carbon, vol. 39, no. 1, pp. 150152, 2001.

[48] D. Liu, Y. Dong, Y. Liu, N. Ma, and G. Sui, "Cellulose nanowhisker (CNW)/graphene nanoplatelet (GN) composite films with simultaneously enhanced thermal, electrical and mechanical properties," Frontiers in Materials, vol. 6, pp. 235-246, 2019. 